\title{
Cosmic distance duality relation and the shape of galaxy clusters
}

\author{
R. F. L. Holanda ${ }^{1}$, J. A. S. Lima ${ }^{1}$, and M. B. Ribeiro ${ }^{2}$ \\ 1 Departamento de Astronomia, Instituto Astronômico e Geofísico, Universidade de São Paulo - USP, São Paulo, Brazil \\ e-mail: [holanda; limajas] @astro.iag.usp.br \\ 2 Instituto de Física, Universidade Federal do Rio de Janeiro - UFRJ, Rio de Janeiro, Brazil \\ e-mail: mbr@if.ufrj.br
}

Received 8 August 2010 / Accepted 24 January 2011

\begin{abstract}
Context. Observations in the cosmological domain are heavily dependent on the validity of the cosmic distance-duality (DD) relation, $\eta=D_{\mathrm{L}}(z)(1+z)^{2} / D_{\mathrm{A}}(z)=1$, an exact result required by the Etherington reciprocity theorem where $D_{\mathrm{L}}(z)$ and $D_{\mathrm{A}}(z)$ are, respectively, the luminosity and angular diameter distances. In the limit of very small redshifts $D_{\mathrm{A}}(z)=D_{\mathrm{L}}(z)$ and this ratio is trivially satisfied. Measurements of Sunyaev-Zeldovich effect (SZE) and X-rays combined with the DD relation have been used to determine $D_{\mathrm{A}}(z)$ from galaxy clusters. This combination offers the possibility of testing the validity of the DD relation, as well as determining which physical processes occur in galaxy clusters via their shapes.

Aims. We use WMAP (7 years) results by fixing the conventional $\Lambda \mathrm{CDM}$ model to verify the consistence between the validity of DD relation and different assumptions about galaxy cluster geometries usually adopted in the literature.

Methods. We assume that $\eta$ is a function of the redshift parametrized by two different relations: $\eta(z)=1+\eta_{0} z$, and $\eta(z)=1+\eta_{0} z /(1+z)$, where $\eta_{0}$ is a constant parameter quantifying the possible departure from the strict validity of the DD relation. In order to determine the probability density function (PDF) of $\eta_{0}$, we consider the angular diameter distances from galaxy clusters recently studied by two different groups by assuming elliptical (isothermal) and spherical (non-isothermal) $\beta$ models. The strict validity of the DD relation will occur only if the maximum value of $\eta_{0}$ PDF is centered on $\eta_{0}=0$.

Results. It was found that the elliptical $\beta$ model is in good agreement with the data, showing no violation of the DD relation (PDF peaked close to $\eta_{0}=0$ at $1 \sigma$ ), while the spherical (non-isothermal) one is only marginally compatible at $3 \sigma$.

Conclusions. The present results derived by combining the SZE and X-ray surface brightness data from galaxy clusters with the latest WMAP results (7-years) favors the elliptical geometry for galaxy clusters. It is remarkable that a local property like the geometry of galaxy clusters might be constrained by a global argument provided by the cosmic DD relation.
\end{abstract}

Key words. X-rays: galaxies: clusters - distance scale - cosmic background radiation

\section{Introduction}

The most useful distances in cosmology are the luminosity distance, $D_{\mathrm{L}}(z)$, and the angular-diameter distance, $D_{\mathrm{A}}(z)$. The expressions of both distances depend on the world models, but the relationship between them, namely

$\frac{D_{\mathrm{L}}}{D_{\mathrm{A}}}(1+z)^{-2}=1$

is valid for arbitrary spacetimes, a result usually referred to as distance-duality (DD) relation.

The above expression can easily be deduced in the context of Friedmann-Robertson-Walker (FRW) cosmologies (Weinberg 1972). However, as originally proven by Etherington (1933), it depends neither on Einstein field equations nor the nature of matter content filling the spacetime. The proof depends crucially on photon conservation (transparency of the cosmic medium) and that sources and observers are linked by null geodesics in a Riemannian spacetime.

The DD relation plays an essential role ranging from gravitational lensing studies to analyses of the cosmic microwave blackbody radiation (CMBR) observations, as well as for galaxy and galaxy cluster observations (Schneider et al. 1992; Komatsu et al. 2011; Lima et al. 2003; Cunha et al. 2007b;
Ribeiro 1992, 2005; Ribeiro \& Stoeger 2003). Indeed, any observational deviation from Eq. (1) would be a theoretical catastrophe thereby igniting a major crises in observational cosmology (Ellis 1971, 2007).

Although taken for granted in virtually all analyses in cosmology, the DD relation is in principle testable by means of astronomical observations. One may assume a redshift dependence of the form

$\frac{D_{\mathrm{L}}}{D_{\mathrm{A}}}(1+z)^{-2}=\eta(z)$

where $\eta(z)$ quantifies a possible epoch-dependent departure from the standard photon conserving scenario $(\eta=1)$.

Basset \& Kuns (2004) used both supernovae Ia data as measurements of the luminosity distance $D_{\mathrm{L}}$ and the estimated $D_{\mathrm{A}}$ of FRIIb radio galaxies (Daly \& Djorgovski 2003) and ultra compact radio sources (Gurvitz 1994, 1999; Lima \& Alcaniz 2000, 2002; Santos \& Lima 2008) in adopting this kind of approach to test possible new physics. Any source of attenuation ("gray" intergalactic dust) or exotic photon interaction must violate the DD relation (More et al. 2009; Avgoustidis et al. 2010), thereby providing new consistency checks of cosmological models.

On the other hand, observations of the Sunyaev-Zeldovich effect (SZE) from galaxy clusters are becoming a powerful tool 
in cosmology (Sunyaev \& Zeldovich 1972; Cavaliere \& FuscoFermiano 1978; De Filippis et al. 2005; Cunha et al. 2007b; Nord et al. 2009; Basu et al. 2010). The combination of SZE and $\mathrm{X}$-ray provides the $D_{\mathrm{A}}(z)$ of galaxy clusters, hence can be used to constrain some cosmological parameters. However, Uzan et al. (2004) argued that this technique is strongly dependent on the validity of the DD relation. When the DD relation does not hold $(\eta \neq 1)$, the observationally determined angular distance must be replaced by the value (in the Uzan et al. (2004) notation the correcting term is $\eta^{-2}$ )

$D_{\mathrm{A}}^{\text {data }}(z)=D_{\mathrm{A}}(z) \eta^{2}$,

this quantity reduces to the conventional angular diameter distance (assuming cosmic transparency) only when the DD relation is strictly valid $(\eta=1)$. To quantify the $\eta$ parameter, Uzan et al. (2004) obtained $D_{\mathrm{A}}(z)$ as given by the cosmic concordance model (Spergel et al. 2003), whereas for $D_{\mathrm{A}}^{\text {data }}(z)$ they considered 18 angular diameters from the Reese et al. (2002) galaxy cluster sample for which a spherically symmetric cluster geometry was assumed. By assuming $\eta$ to be constant, their statistical analysis provided $\eta=0.91_{-0.04}^{+0.04}(1 \sigma)$ and is therefore only marginally consistent with the standard result, $\eta=1$.

De Bernardis et al. (2006) also searched for deviations from the DD relation by using the $D_{\mathrm{A}}^{\text {data }}(z)$ from galaxy clusters provided by the sample of Bonamente et al. (2006). They found a non violation of the DD relation in the framework of the cosmic concordance $\Lambda$ CDM model. Avgoustidis et al. (2010) adopted a DD relation of the form $D_{\mathrm{L}}=D_{\mathrm{A}}(1+z)^{2+\epsilon}$ to constrain the cosmic opacity by combining the SN Type Ia data compilation of Kowalski et al. (2008) with the latest measurements of the Hubble expansion at redshifts in the range $0<z<2$ (Stern et al. 2010). By working in the context of a flat $\Lambda$ CDM model, they found $\epsilon=-0.04_{-0.07}^{+0.08}(2 \sigma)$.

In the past few years, many studies based on Chandra and $X M M$ observations have shown that in general galaxy clusters exhibit elliptical surface brightness maps. Simulations have also predicted that dark matter halos show axis ratios typically of the order of $\approx 0.8$ (Wang \& White 2009), thereby disproving the spherical geometry assumption usually adopted (Reiprich \& Boringer 2002; Bonamente et al. 2006; Shang et al. 2009). In this line, the first determination of the intrinsic threedimensional (3D) shapes of galaxy clusters was presented by Morandi et al. (2010) by combining X-ray, weak-lensing, and strong-lensing observations. They studied the galaxy cluster MACS J1423.8+2404 and found a tri-axial galaxy cluster geometry with DM halo axial ratios $1.53 \pm 0.15$ and $1.44 \pm 0.07$ on the plane of the sky and along the line of sight, respectively.

In this Letter, we take the validity of the DD relation for granted to access the galaxy cluster morphology. The values of $D_{\mathrm{A}}(z)$ are obtained from the WMAP (7 years) results by fixing the conventional flat $\Lambda \mathrm{CDM}$ model whereas the observational measurements $D_{\mathrm{A}}^{\text {data }}(z)$ are the angular diameter distances from galaxy clusters obtained via SZE plus X-ray techniques. These samples differ in terms of the assumptions concerning the possible cluster geometries of elliptical and spherical models. Our analysis is based on two parametric representations of $\eta(z)$ defined by Eq. (2) (or Eq. (3)), namely

$$
\text { I. } \eta(z)=1+\eta_{0} z \quad \text { and } \quad \text { II. } \eta(z)=1+\eta_{0} z /(1+z) \text {. }
$$

The first expression is a continuous and smooth one-parameter linear expansion, whereas the second one includes a possible epoch-dependent correction, which avoids the divergence at extremely high $z$. These deformations of the DD relation effectively parametrize our ignorance of the underlying process responsible for its possible violation. However, we emphasize that these expressions are very simple and have several advantages such as a manageable one-dimensional phase space and a good sensitivity to observational data. Clearly, the second parametrization can also be rewritten as $\eta(z)=1+\eta_{0}(1-a)$, where $a(z)=(1+z)^{-1}$ is the cosmic scale factor. This represents an improvement with respect to the linear parametrization, since the DD relation becomes bounded regardless of the redshift values. It will become more useful once higher redshift clusters data become available.

The above parametrizations are clearly inspired by similar expressions for the $\omega(z)$-equation of state parameter of dark energy models (Padmanabhan \& Choudury 2003; Linder 2003; Cunha et al. 2007a; Silva et al. 2007). In the limit of extremely low redshifts $(z \ll 1)$, we have $\eta=1$ and $D_{\mathrm{L}}=D_{\mathrm{A}}$ as should be expected, and, more important for our subsequent analysis, the value $\eta_{0}=0$ must be favored by the Etherington result. In other words, for a given data set, the likelihood of $\eta_{0}$ must peak at $\eta_{0}=0$ to satisfy the cosmic relation. As we shall see, for those accepting the strict validity of the standard DD relation, our analysis suggests that galaxy clusters have an elliptical geometry. In principle, this kind of result is an interesting example of how a cosmological (global) condition correlates with the local physics.

\section{Galaxy cluster samples}

To constrain the values of $\eta_{0}$, we consider two galaxy cluster samples for which $D_{\mathrm{A}}(z)^{\text {data }}$ were derived by combining their SZE and X-ray surface brightness observations.

The first sample is formed by 25 galaxy clusters compiled by De Filippis et al. (2005). By using an isothermal elliptical $\beta$ model to describe the clusters, the $D_{\mathrm{A}}(z)^{\text {data }}$ was derived for two subsamples discussed in the literature. The first one, compiled by Reese et al. (2002), is a selection of 18 galaxy clusters distributed over the redshift interval $0.14<z<0.8$. The second subsample of Mason et al. (2001) has 7 clusters from the X-ray limited flux sample of Ebeling et al. (1996). These 25 pieces of data are referred to as the elliptical sample.

The second sample is defined by the 38 galaxy clusters observed by Bonamente et al. (2006), where the cluster plasma and dark matter distributions were analyzed assuming hydrostatic equilibrium model and spherical symmetry. This sample consists of clusters that have both X-ray data from the Chandra Observatory and SZE data from the BIMA/OVRO SZE imaging project, which uses the Berkeley-Illinois-Maryland Association (BIMA) and Owens Valley radio observatory (OVRO) interferometers to image the SZE. This dataset is termed the spherical sample.

In Fig. 1, we plot the elliptical and spherical galaxy cluster samples. Some authors have adopted these samples to estimate the galaxy cluster distances and measure the Hubble parameter by means of the SZE/X-ray technique (Bonamente et al. 2006; Cunha et al. 2007b). However, since these samples are endowed with different geometric assumptions, our main interest here is to confront these underlying hypotheses with the validity of the DD relation.

At present, there is no convincing evidence for deviations from the minimal cosmic concordance flat model (Komatsu et al. 2011, Percival et al. 2010; Riess et al. 2009); In this case, the angular diameter distance is given by Lima et al. (2003) and 
R. F. L. Holanda et al.: Cosmic distance duality relation and the shape of galaxy clusters

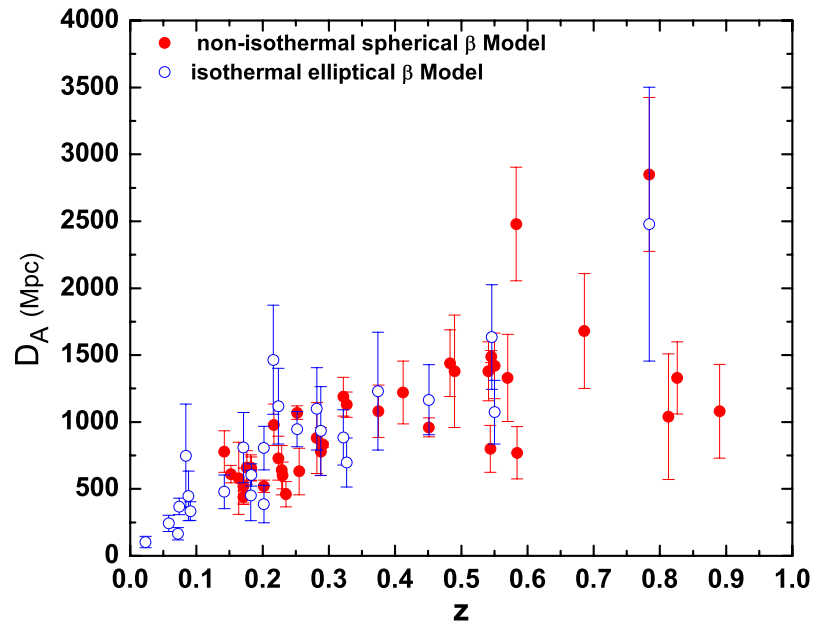

Fig. 1. Galaxy clusters data. The open (blue) and filled (red) circles with the associated error bars represent, respectively, the De Filippis et al. (2005) and Bonamente et al. (2006) samples.

Cunha et al. (2007b)

$D_{\mathrm{A}}\left(z ; h, \Omega_{\mathrm{m}}\right)=\frac{3000 h^{-1}}{(1+z)} \int_{0}^{z} \frac{\mathrm{d} z^{\prime}}{\mathcal{H}\left(z^{\prime} ; \Omega_{\mathrm{m}}\right)} \mathrm{Mpc}$,

where $h=H_{0} / 100 \mathrm{~km} \mathrm{~s}^{-1} \mathrm{Mpc}^{-1}$ and the dimensionless function $\mathcal{H}\left(z^{\prime} ; \Omega_{\mathrm{m}}\right)$ is given by

$\mathcal{H}=\left[\Omega_{\mathrm{m}}\left(1+z^{\prime}\right)^{3}+\left(1-\Omega_{\mathrm{m}}\right)\right]^{1 / 2}$.

In our statistical analysis (see next section), the $D_{\mathrm{A}}(z)$ value was calculated by using the cosmological parameters given in the joint analysis carried out by Komatsu et al. (2011), namely $\Omega_{\Lambda}=\left(1-\Omega_{\mathrm{m}}\right)=0.728 \pm 0.015$ and $h=0.704 \pm 0.013$.

\section{Analysis and results}

We estimate the $\eta_{0}$ parameter for each sample and both parameterizations of $\eta(z)$, namely, $\eta(z)=1+\eta_{0} z$ and $\eta(z)=$ $1+\eta_{0} z /(1+z)$. To begin with, we evaluate the likelihood distribution function $\mathrm{e}^{-\chi^{2} / 2}$, where

$\chi^{2}=\sum_{z} \frac{\left[\eta^{2}(z)-\eta_{\mathrm{obs}}^{2}(z)\right]^{2}}{\sigma_{\eta_{\mathrm{obs}}^{2}}}$

and $\eta_{\mathrm{obs}}^{2}(z)=D_{\mathrm{A}}^{\text {data }}(z) / D_{\mathrm{A}}(z)$ (see Eq. (3)). The statistical and systematic errors have been discussed by many authors (Mason et al. 2001; Reese et al. 2002, 2004; Bonamente et al. 2006). Statistical error contributions for galaxy clusters are: SZE point sources $\pm 8 \%$, X-ray background $\pm 2 \%$, Galactic $N_{\mathrm{H}} \leq \pm 1 \%$, $\pm 15 \%$ for cluster asphericity, $\pm 8 \%$ kinetic SZ and for CMBR anisotropy $\leq \pm 2 \%$. On the other hand, the estimates of systematic effects are: SZ calibration $\pm 8 \%, \mathrm{X}$-ray flux calibration $\pm 5 \%$, radio halos $+3 \%$, and $X$-ray temperature calibration $\pm 7.5 \%$. One may show that typical statistical errors can reach nearly $20 \%$, in agreement with other works (Mason et al. 2001; Reese et al. $2002,2004)$, whereas for systematics we also find typical errors around $+12.4 \%$ and $-12 \%$ (see also Table 3 in Bonamente et al. 2006).

In Fig. 2, we plot the likelihood distribution function for each galaxy cluster data. The basic results are:

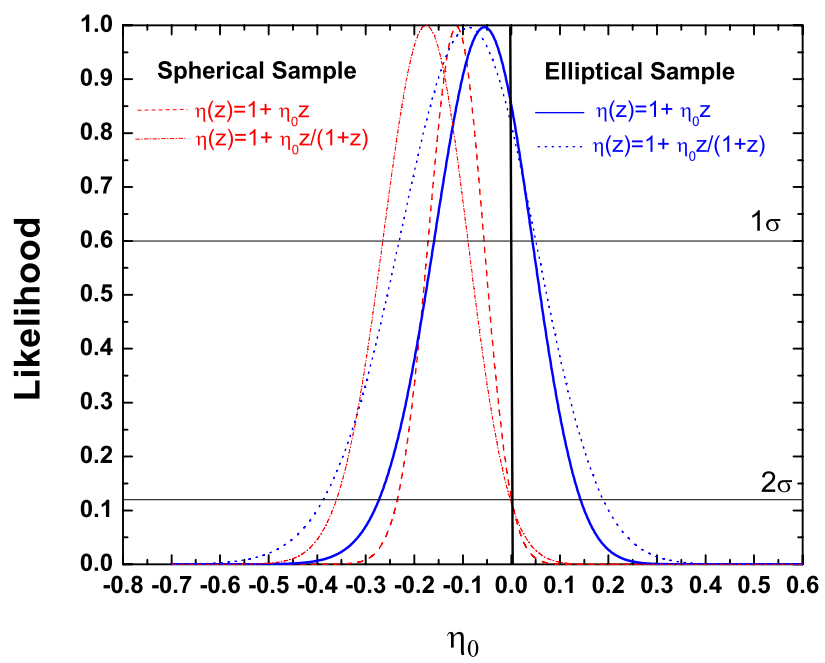

Fig. 2. The likelihood distribution functions for spherical (dotteddashed and dashed red lines) and elliptical (dotted and solid blue lines) cases. See the text for values of $\eta_{0}$.

- For the elliptical sample, we obtain $\eta_{0}=-0.056_{-0.1}^{+0.1}\left(\chi_{\text {d.o.f. }}^{2}=\right.$ $0.98)$ and $\eta_{0}=-0.088_{-0.14}^{+0.14}\left(\chi_{\text {d.o.f. }}^{2}=0.97\right)$ in $68 \%$ c.l. for a linear (blue solid line) and non linear (blue dotted line) parametrization, respectively.

- For the spherical sample, we obtain $\eta_{0}=-0.12_{-0.055}^{+0.055}$ $\left(\chi_{\text {d.o.f. }}^{2}=0.85\right)$ and $\eta_{0}=-0.175_{-0.083}^{+0.083}\left(\chi_{\text {d.o.f. }}^{2}=0.84\right)$ in $68 \%$ c.l. for a linear (red dashed line) and non linear (red dotted dashed line) parametrization.

The uncertainties in $\eta_{0}$ include (in quadrature) the systematic plus statistical errors. We can see that the $D_{\mathrm{A}}^{\text {data }}(z)$ inferred based on the elliptical description agrees with the DD relation, whereas the other case, in which a spherical $\beta$ model was assumed to describe the clusters, is only marginally compatible with the DD relation. This result remains valid even when only clusters with $z>0.1$ are considered in the elliptical sample. In this situation, we obtain $\eta_{0}=-0.044_{-0.1}^{+0.1}\left(\chi_{\text {d.o.f. }}^{2}=0.94\right)$ for the linear parametrization, and $\eta_{0}=-0.07_{-0.14}^{+0.14}\left(\chi_{\text {d.o.f. }}^{2}=0.93\right)$ within $1 \sigma$ in the non-linear case.

It thus follows that we have found no evidence of violation of the DD relation when the elliptical case is considered. However, the same kind of analysis shown in Fig. 2 points to a contradiction with the spherical symmetry hypothesis assumed in the Bonamente et al. (2006) sample.

\section{Conclusions}

We have explored some consequences of a deformed distance duality relation, $\eta(z)=D_{\mathrm{L}}(1+z)^{-2} / D_{\mathrm{A}}$, based on observations of Sunyaev-Zeldovich effect and X-ray from galaxy clusters. The consistency between the strict validity of the standard relation $(\eta(z) \equiv 1)$ and the assumptions regarding the geometry used to describe the galaxy clusters (elliptical and spherical $\beta$ models) has been discussed. The $\eta(z)$ function was parametrized in two distinct forms, $\eta=1+\eta_{0} z$ and $\eta=1+\eta_{0} z /(1+z)$, where $\eta_{0}$ is a constant parameter quantifying a possible departure from the strict validity of the duality relation. The basic idea pursued in this work is a simple one. The likelihood of the free parameter appearing in the proposed expressions for $\eta(z)$ should 
peak around $\eta_{0}=0$ when the distance duality relation is strictly obeyed.

By comparing the De Filippis et al. (2005) (elliptical isothermal $\beta$ model) and Bonamente et al. (2006) (spherical nonisothermal $\beta$ model) samples with $D_{\mathrm{A}}(z)$ obtained from $\Lambda \mathrm{CDM}$ (WMAP7), we show that the elliptical geometry is more consistent with no violation of the duality relation. The uncertainties in $\eta_{0}$ included the systematic plus statistical errors from cluster data. In the case of an elliptical sample (see Fig. 2), we found that $\eta_{0}=-0.056_{-0.1}^{+0.1}$ and $\eta_{0}=-0.088_{-0.14}^{+0.14}$ for the linear and non-linear parametrization, respectively. However, the spherical sample (see Fig. 2) is only marginally compatible with $\eta_{0}=-0.12_{-0.055}^{+0.055}$ and $\eta_{0}=-0.175_{-0.083}^{+0.083}$ for linear and non-linear parametrization, respectively. Our analysis reveals that the elliptical model is compatible with the duality relation validity at $1 \sigma$, whereas the spherical model is only marginally compatible at $3 \sigma$.

At this point, it is interesting to compare our results with those obtained by following a complementary approach (Holanda et al. 2010). The $\eta(z)$ function there was also parametrized as in the present work. However, the overall discussion was based on a model-independent cosmological test by considering $D_{\mathrm{A}}(z)$ from galaxy clusters and the luminosity distances given by two sub-samples of $\mathrm{SNe}$ Ia taken from the constitution data (Hicken et al. 2009). Both analyse are consistent with each other and suggest that the elliptical model is more compatible with the validity of the standard duality relation than the spherical case.

Summarizing, the statistical analysis presented here provides additional evidence that the true geometry of clusters has an elliptical form. In principle, it is remarkable that a local property (the geometry of galaxy clusters) might be constrained by a global argument such as the one provided by the cosmological distance duality relation. In the near future, as more and larger data sets with smaller statistical and systematic uncertainties become available, the method proposed here (based on the validity of the distance duality relation) can improve the limits on the measurements of cluster geometries.

Acknowledgements. The authors are grateful to an anonymous referee for helpful comments and suggestions that improved the original version of the work. We also thank Antonio Guimarães for helpful discussions. R.F.L.H. is supported by FAPESP (No. 07/52912-2), J.A.S.L. is partially supported by CNPq (No. 304792/2003-9) and FAPESP (No. 04/13668-0) and MBR is partially supported by FAPERJ.

\section{References}

Avgoustidis, A., Burrage, C., Redondo, J., Verde, L., \& Jimenez, R. 2010, JCAP, 1010, 024

Basset, B.A., \& Kunz, M. 2004, PRD, 69, 101305

Basu, K., Zhang, Y.-Y., Sommer, M. W., et al. 2010, A\&A, 519, A29

Bonamente, M., Chapman, S. C., Ibata, R. A., et al. 2006, ApJ, 647, 25

Cavaliere, A., \& Fusco-Fermiano, R. 1978, A\&A, 667, 70

Cunha, J. V., Marassi, L., \& Santos, R.C. 2007a, IJMPD, 16, 403

Cunha, J. V., Marassi, L., \& Lima, J. A. S. 2007b, MNRAS, 379, L1

Daly, R. A., \& Djorgovski, S. G. 2003, ApJ, 597, 9

De Bernardis, F., Giusarma, E., \& Melchiorri A. 2006, IJMPD, 15, 759

De Filippis, E., Sereno, M., Bautz, M. W., \& Longo G. 2005, ApJ, 625, 108

Ebeling, H., Voges, W., Bohringer, H., et al. 1996, MNRAS, 281, 799

Ellis, G. F. R. 1971, Relativistic Cosmology, Proc. Int. School Phys. Enrico Fermi, ed. R. K. Sachs (New York: Academic Press), 104; reprinted in 2009, GRG, 41, 581

Ellis, G. F. R. 2007, GRG, 39, 1047

Etherington, I. M. H. 1933, Phil. Mag., 15, 761; reprinted in 2007, GRG, 39, 1055

Gurvitz, L. I. 1994, ApJ, 425, 442

Gurvitz, L. I., Kellermann, K. I., \& Frey, S. 1999, A\&A, 342, 378

Hicken, M., Wood-Vasey, W. M., Blondin, S., et al. 2009, ApJ, 700, 1097

Holanda, R. F. L., Lima, J. A. S., \& Ribeiro, M. B. 2010, ApJ, 722, L233

Kowalski, M., Rubin, D., Aldering, G., et al. 2008, ApJ, 686, 749

Komatsu, E., Smith, K. M., Dunkley, J., et al. (WMAP collaboration) 2011, ApJS, 192, 18

Lima, J. A. S., \& Alcaniz, J. S. 2000, A\&A, 357, 393

Lima, J. A. S., \& Alcaniz, J. S. 2002, ApJ, 566, 15

Lima, J. A. S., Cunha, J. V., \& Alcaniz, J. S. 2003, Phys. Rev. D, 68, 023510

Linder, E. V. 2003, PRL, 90, 091301

More, S., Bovy, J., \& Hogg, D. W. 2009, ApJ, 696, 1727

Mason, B. S., Myers, S. T., \& Readhead, A. C. S. 2001, ApJ, 555, L11

Morandi, A., Pedersen, K., \& Limousin, M. 2010, ApJ, 713, 491

Nord, M., Basu, K., Pacaud, F., et al. 2009, A\&A, 506, 623

Padmanabhan, T., \& Choudury R. 2003, MNRAS, 344, 823

Percival, W., et al. 2010, MNRAS, 401, 2148

Reiprich, T. H., \& Bohringer, H. 2002, ApJ, 567, 716

Ribeiro, M. B. 1992, ApJ, 388, 1

Ribeiro, M. B. 2005, A\&A., 429, 65

Ribeiro, M. B., \& Stoeger, W. R. 2003, ApJ, 592, 1

Reese, E. D. 2004, in Measuring and Modeling the Universe, ed. W. L. Freedman (CUP), 138

Reese, E. D., Carlstrom, J. E., Joy, M., et al. 2002, ApJ, 581, 53

Riess, A., et al. 2009, ApJ, 116, 1009

Santos, R. C., \& Lima, J. A. S. 2008, PRD, 77, 083505

Schneider, P., Ehlers, J., \& Falco, E. E. 1992 Gravitational Lenses (Berlin: Springer-Verlag)

Shang, C., Haiman, Z., \& Verde, L. 2009, MNRAS, 400, 1085

Silva, R., Alcaniz, J. S., \& Lima, J. A. S. 2007, IJMPD, 16, 469

Spergel, D. N., Verde, L., Peiris, H. V., et al. 2003, ApJS, 148, 175

Stern, D., Jimenez, R., Verde, L., Kamionkowski, M. \& Stanford, S. A. 2010, JCAP, 02,008

Sunyaev, R. A., \& Zel'dovich Ya. B. 1972, Comm. Astrophys. Space Phys., 4, 173

Uzan, J. P., Aghanim, N., \& Mellier Y. 2004, PRD, 70, 083533

Wang, J., \& White, S. D. M. 2009, MNRAS, 396, 709

Weinberg, S. 1972, Gravitation and Cosmology (New York: Wiley) 